\title{
La práctica pedagógica del docente de enfermería: del conductismo al constructivismo
}

\author{
Claudia María Moreno Mojica ${ }^{1}$, Julián Andrés Barragán Becerra \\ Universidad Pedagógica y Tecnológica de Colombia - Colombia
}

Autor de correspondencia: ${ }^{1}$ claudiamaria.moreno@uptc.edu.co

Recibido: 25 de agosto de 2019 Revisado: 07 de noviembre de 2019 Aprobado: 18 de marzo de 2020 Publicado: 18 de junio de 2020

\section{Resumen}

El objetivo fue identificar las prácticas pedagógicas de los docentes de un programa de enfermería y su posible relación con el modelo constructivista. El enfoque es cualitativo y fenomenológico, a partir del estudio de caso único, el cual permitió conocer la complejidad de la situación investigada dentro de un programa académico universitario. Se utilizaron la observación y la cartografía social pedagógica como técnicas de correlación. Surgieron las categorías y subcategorías deductivas: mediador maestro- y enseñar - escenario didáctico, el estudiante y estrategias pedagógicas-. Se identificó que las prácticas pedagógicas de los docentes confluyen en el modelo tradicional de transmisión de conocimientos, sobre las que realizan un proceso reflexivo para ser coherentes con el modelo pedagógico constructivista que propone el programa.

Palabras clave: pedagogía, enseñanza, aprendizaje, modelo educacional, enfermería

\section{(c) $(1) \Theta$}

Para citar este artículo: Moreno, C., \& Barragan, J. (2020). La práctica pedagógica del docente de enfermería: del conductismo al constructivismo. Praxis \& Saber, 11(26), e10255. https://doi. org/10.19053/22160159.v11.n26.2020.10255 


\title{
The pedagogical practice of the nursing care teacher: from behaviorism to constructivism
}

\begin{abstract}
The objective was to identify the pedagogical practices of teachers in a nursing programme and their possible relation to the constructivist model. The approach is qualitative and phenomenological, based on the single case study, which provided insight into the complexity of the situation investigated in a university academic programme. Observation and pedagogical social cartography were used as correlation techniques. The following deductive categories and subcategories emerged: mediator-teacherand teaching-didactic scenario, the student and pedagogical strategies. It was identified that the teachers' pedagogical practices converge in the traditional model of knowledge transmission, on which they carry out a reflexive process in order to be coherent with the constructivist pedagogical model proposed by the programme.
\end{abstract}

Keywords: pedagogy, teaching, learning, educational model, nursing

\section{A prática pedagógica do docente de enfermagem: do behaviorismo ao construtivismo}

\section{Resumo}

O objetivo foi identificar as práticas pedagógicas dos docentes de um programa de enfermagem e sua possível relação com o modelo construtivista. O enfoque é qualitativo e fenomenológico, a partir do estudo de caso único, o qual permitiu conhecer a complexidade da situação pesquisada dentro de um programa acadêmico universitário. Utilizaram-se a observação e a cartografia social pedagógica como técnicas de correlação. Surgiram as categorias e subcategorias dedutivas: mediador - docente - e ensino - espaço didático, o aluno e estratégias pedagógicas. Identificou-se que as práticas pedagógicas dos docentes convergem no modelo tradicional de transmissão de conhecimentos, sobre as que realizam um processo reflexivo para ser coerentes com o modelo pedagógico construtivista que propõe o programa.

Palavras-chave: pedagogia, ensino, aprendizagem, modelo educacional, enfermagem

\section{Introducción ${ }^{1}$}

Por más de un siglo, la práctica pedagógica en enfermería se estableció a partir del modelo biomédico, que no responde a las necesidades humanistas que plantea la educación actual. Es un reto para el docente repensar y reflexionar si el modelo pedagógico tradicional

1. Artículo de investigación científica y tecnológica, sin financiación. Proyecto: La práctica pedagógica del docente de enfermería: del conductismo al constructivismo. 
conductista sobre el que realiza la formación profesional logra potenciar capacidades, habilidades, competencias cognoscitivas y emocionales en un estudiante reconocido como un ser único, autónomo y responsable de su aprendizaje (Noreña, Alcaraz, Amador \& Ramos, 2013).

El sistema educativo impulsa el constructivismo a partir de modelos, metodologías y estrategias, para la formación integral del enfermero. En Colombia, la reforma permanente de los planes de estudio y de los currículos busca dar respuesta a esta tendencia, que implica abandonar la postura reduccionista del transmisor-receptor del conocimiento, para reconocer a la persona en el contexto del proceso de enseñanza-aprendizaje desde la mediación y acompañamiento como eje central de la práctica pedagógica (Paim, lappe \& Rocha, 2015).

Porlán (1987) y Sacristán (1991) comprenden la práctica pedagógica como una acción dotada de sentido y de razones que permiten comprender, innovar, investigar, profundizar y transformar el proceso de enseñanza-aprendizaje, al crear condiciones adecuadas para que el estudiante, en interacción con el maestro como mediador, estimule el pensamiento creativo, crítico y reflexivo en la autoconstrucción del conocimiento. Desde la realidad en el aula, se crean herramientas y situaciones conjuntas a partir de variables contextuales que llevan al diálogo, la libertad y autonomía de los actores, que son reconocidos como seres singulares dentro de una sociedad y cultura que pretenden transformar, desde la formación integral, el desarrollo humano y los cambios emergentes (Flórez, Mayorga \& Vargas, 2017).

El desarrollo de este concepto en los programas de enfermería permite transitar de la techné a la phrónesis desde el acto reflexivo, consciente y prudente del compromiso que tiene el enfermero como docente mediador, capaz de dignificar, abrir oportunidades, compartir y escuchar en el contexto del estudiante y de contar con experiencia teórica, metodológica y práctica para desarrollar el proceso de enseñanza en un currículo que, según Martín y Porlán (citados por García, 2005, p 69), "permita desaprender para aprender de una manera activa y experimental, para promover en el estudiante el desarrollo de sus propias estrategias cognoscitivas al sentirse sujeto actuante de su proceso de aprendizaje".

En el plan de estudios de un programa de enfermería, se define el proceso de enseñanzaaprendizaje desde un modelo constructivista y holístico, opuesto al modelo tradicional, que abandona la perspectiva racionalista y positivista. Por lo tanto, se pretendió identificar la diversidad de prácticas pedagógicas docentes y su posible relación con una visión constructivista desde el desarrollo de pensamiento crítico y reflexivo, en el que se comprende la realidad y las necesidades del estudiante, así como las del ser maestro, al promover la construcción del aprendizaje (Agama \& Knopfler, 2016). El estudio generó procesos de reflexión continua en el docente frente a prácticas pedagógicas y la coherencia con nuevos modelos constructivistas que desarrollan en el estudiante la inteligencia cognitiva y emocional a través de la cooperación y la comunicación efectiva (Castro \& Pereira, 2011).

\section{Material y métodos}

El estudio cualitativo con enfoque fenomenológico permitió estudiar y conocer la esencia de la experiencia humana vivida, definida por Prado, Souza, Monticelli, Cometto y 
Gómez (2013) como "un movimiento cuyo objetivo es la descripción de los fenómenos que se experimentan de manera consciente, libre de supuestos y prejuicios" (p. 13).

El estudio de caso único como método permitió conocer la complejidad de una situación particular dentro del contexto de vida real (Stake, 2007). Se identificaron las prácticas pedagógicas de los docentes de un programa de enfermería de Boyacá y su posible relación con el modelo constructivista. Este es un caso instrumental, ya que se eligió por ser prototípico. Se pretendió identificar a profundidad algunas actividades, respuestas o problemas.

La recolección de información se hizo con ayuda de dos técnicas de investigación. La primera es la observación, mediante la cual el investigador hace uso de sus sentidos e intenta comprender el fenómeno de estudio a partir de la realidad de los participantes, del cual emergen aspectos significativos de análisis que orientan observaciones subsiguientes (Burns \& Grove, 2004). La segunda es la cartografía social pedagógica, con la que el investigador acompaña al grupo en la reflexión sobre sus prácticas de enseñanza y aprendizaje, mediante la creación de un mapa en el que se evidencian las problemáticas del territorio - aula, universidad- (Barragán \& Amador, 2014, p. 137).

\section{Contexto y participantes}

Para la fase de observación, se eligieron un grupo de tercer semestre - de 28 estudiantes y 3 docentes - y un grupo de sexto semestre - de 19 estudiantes y 2 docentes-, debido a que son semestres intermedios que permiten una comparación inicial y media del plan de estudios, así como la disponibilidad en las aulas. Las asignaturas tenían una intensidad semanal alta y fueron desarrolladas a través de módulos o contenidos temáticos. La frecuencia de la observación estuvo relacionada con el módulo o contenido temático seleccionado. Se realizó observación durante dos semanas.

Para la fase de cartografía, se hizo un taller con doce docentes del programa con vinculación plena y mayor a un año, quienes diseñaron cinco mapas colectivos en línea del tiempo. Se presentaron los objetivos del estudio y la metodología. Se llevó a cabo el diligenciamiento del consentimiento informado y la participación autónoma y voluntaria, como responsabilidad ética. Se respetaron los principios de: confidencialidad de la información; de no maleficencia, respecto a posibles consecuencias de la participación; y de beneficencia hacia el aporte a los procesos académicos de los grupos y del programa como mejoramiento. Estos principios están contenidos en el Código deontológico de enfermería en Colombia (Ley 911, 2004).

\section{Criterios de recolección, análisis y sistematización}

La vinculación de los participantes y la recolección de los datos se realizaron entre agosto y noviembre del 2016, los cuales se identificaron a través de la dirección del programa. Se obtuvo aprobación del comité curricular.

El análisis se realizó a partir de la transcripción y lectura de rejillas de observación, audios y material escrito, mapas de los talleres de cartografías y de todas las descripciones de los participantes, orales y escritas. El fin de las descripciones era tener un sentimiento frente a ellas y extraer enunciados significativos. Los temas se subrayaron y se consignaron en la matriz de análisis, para encontrar interpretaciones a partir de códigos. La elaboración 
de memos y temas se organizó en grupos que aportaban significados similares en una experiencia para construir la categorización (Jaramillo \& Aguirre, 2012) y se llevó a cabo el proceso deductivo de codificación, agrupación de códigos en subcategorías y categorías, las afirmaciones registradas se presentan en números de grupo, semestre u observación.

El rigor metodológico se obtuvo a partir de: la credibilidad, que se logró mediante verificación de la información con la retroalimentación a un grupo de estudiantes y docentes; la auditabilidad, con la estructuración de un marco de diseño y análisis del estudio mediante asesoría de grupo de expertos en investigación; y reflexividad, realizada por un experto en pedagogía, con quien se sostuvo una extensa discusión sobre los hallazgos, conclusiones y análisis de los datos a partir de la triangulación (Prado et al., 2013).

\section{Resultados y discusión}

Las categorías y subcategorías que surgieron deductivamente se establecieron desde los teóricos: Benner (citada por Carrillo, García, Wilches, Cárdenas, Díaz, 2013), quien propone el proceso de enseñanza en la formación del profesional de enfermería en relación con prácticas pedagógicas; y Porlán (1987) y Sacristán (2010), para quienes la práctica pedagógica es la mediación que realiza el profesor entre la teoría, la práctica y el proceso de enseñanza-aprendizaje, que pretende desarrollar una visión constructivista del desarrollo humano. Igualmente, el modelo pedagógico holístico y configuracional dialéctico del programa integra el enfoque constructivista e histórico cultural (figura 1).

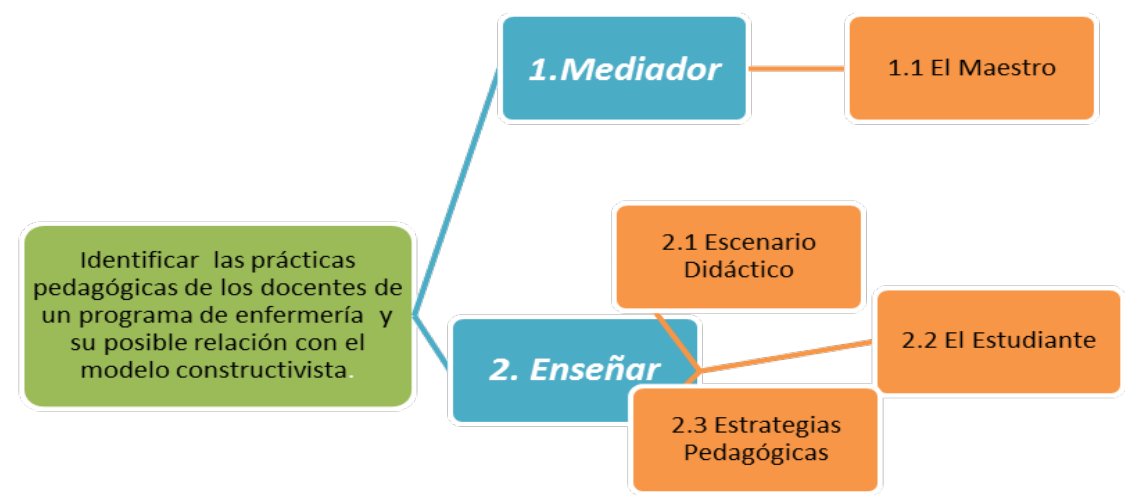

Figura 1. Categorías y subcategorías. Fuente: elaboración propia.

\section{Mediador.}

Según Porlán y Martín (1994), el trabajo del maestro como mediador entre la teoría y la práctica guarda relación con el modelo alternativo de enseñanza basado en una "concepción sistémica y compleja de la realidad, en donde el proceso de enseñanza-aprendizaje pretende una visión constructivista del desarrollo humano" (p. 2). Así, el maestro es quien asume el papel de facilitador e investiga su propia actividad profesional.

Desde la observación de campo y la cartografía social pedagógica [CSP], emerge la figura de maestro, que confluye y discrepa desde las características propuestas por Porlán y Sacristán: "La profesora es quien dispone el salón y propone la forma en que se desarrolla la 
clase. Es ella quien lleva los tiempos y plantea las metodologías. Desde su discurso posibilita o no la participación de los estudiantes" (Grupo 1, semestre 3, observación 1).

Las profesoras realizan una parte de socialización del módulo e involucran todo el grupo de estudiantes en el desarrollo de la clase. Es a partir de casos y situaciones clínicas que, junto al uso de simuladores, lleva a que los estudiantes se muestren activos y participativos en los procedimientos que realizan. (Grupo 3, estudiantes, semestre 3).

El 60\% de docentes de enfermería participantes, como mediadores entre la teoría y la práctica en el aula, mantiene posturas biomédicas y autoritarias que generan conductas esperadas en aprendizajes memorísticos. Limitan la práctica reflexiva y la construcción del conocimiento por parte de los estudiantes (Sánchez, 2001). En contraste, el 40\% de docentes inicia un camino por el constructivismo con propuestas que, aunque integran la trasmisión de conocimiento desde modelos tradicionales, incursionan en prácticas que les permiten ser facilitadores y romper esquemas reduccionistas.

Desde lo que significa ser maestros de enfermería, se presenta el mapa colectivo En construcción y re-ingeniería (imagen 1). En la línea del tiempo, el territorio existencial se representa por la lámpara como símbolo de enfermería. La naturaleza icónica de la imagen representa las prácticas pedagógicas de los docentes en construcción en el presente y en reingeniería en un futuro. Desde el contenido, el indicador azul simboliza al docente actual e investigador que representa ser observado y criticado. Hay un cerebro cansado sobrepuesto, que demuestra el no reconocimiento de su aprendizaje. En la parte inferior aparece el ícono de maestro tradicional, sobre el que escriben "en proceso de reducción". Finalmente, a la derecha, están el pictograma de Mafalda con un libro y cara expresiva que dice "esperan el conocimiento absoluto" y un ícono de pensamiento que representa innovación. En lo expuesto por el grupo de docentes, está la representación de maestro del presente:

En el presente, como docentes, nos vemos como personas en proceso de construcción, que nos sentimos observados por los estudiantes y los compañeros, de una forma positiva para guiarnos y de una forma negativa desde el proceso de estar criticando si lo que hacemos es correcto o no. Para los estudiantes, nosotros debemos tener un conocimiento absoluto para ser considerados docentes. Con los compañeros, sentimos que no somos aún docentes, que nos hace falta mucho para alcanzar las expectativas que ellos tienen y el poder innovar como lo esperan. (Grupo 1, Docentes)

Para Sacristán (2010), "los alumnos saben la singularidad de sus profesores; seguramente son aquellos más acertados cuando los valoran que cuando son valorados por estos" (p. 248). Así, la concepción de maestro en construcción puede ser un camino mediador en la reflexión sobre la práctica docente, la individualidad y vivencia con los factores que inciden en la práctica. Los resultados son coherentes con la necesidad de formación y preparación del enfermero que desea seguir la docencia, ya que sus prácticas pedagógicas siguen un paradigma tradicional, con dificultad para incorporar modelos emergentes, que llevan a la dicotomía entre teoría y práctica, al no facilitar el aprendizaje desde diferentes situaciones problema (Rodrigues, Mendes \& Carvalho, 2008).

El maestro del futuro es aquel que rediseña sus procesos de enseñanza. Está representado por el ícono de un cerebro fuerte que simboliza el conocimiento y por el pictograma de Mafalda que tiene una dimensión mayor a la de sus amigos y la expresión de reclamo. 
El maestro que esperan ser en el futuro es: "un ser reconocido por los compañeros y los estudiantes, para así vernos como unos docentes motivadores, empoderados que ayudemos a los estudiantes" (Grupo 1, docentes).

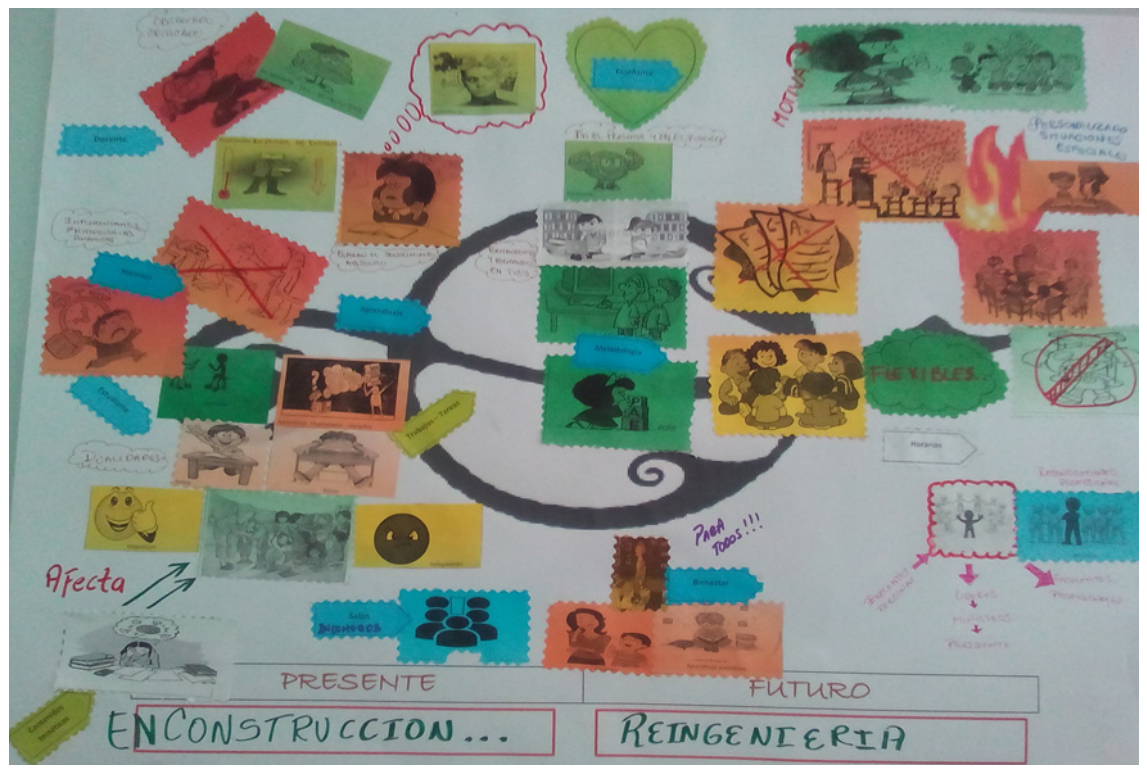

Imagen 1. Mapa: En construcción y re-ingeniería. Fuente: docentes del programa de enfermería.

La imagen de Mafalda sobre el maestro motivador representa una hipérbole de la situación o el tránsito de docente tradicional al mediador constructivista representado desde la percepción de ser maestro en el futuro como: "Facilitador" (Grupo 2, docentes); "ser que se reconozca como igual al estudiante y permita una enseñanza saludable. Para esto debemos eliminar de nuestra mente que lo sabemos todo y que somos quienes damos el conocimiento" (Grupo3, docentes) "ser humano con formación pedagógica y humanista" (Grupo 4, docentes).

\section{Enseñar}

Sacristán (citado por Cadavid \& Calderón), define la enseñanza como:

Un arte en la medida que para ejecutarla cuenta la acción que la conduce, es decir es un proceso de desarrollo, en tanto ofrece direcciones y alternativas para intervenir las cualidades personales de sus agentes, quienes son una selección de la cultura, la sociedad y la ciencia. (2001, p. 148)

\section{Escenario didáctico.}

El aula como escenario didáctico y entorno inmediato del profesor y el estudiante condiciona el proceso de enseñanza-aprendizaje, a partir de la estructura social, juego de roles, relaciones personales y estructura académica en el intercambio de conocimientos y la forma de interpretar la realidad de cada uno de los actores (Porlán, 1987).

En la observación de campo, el escenario didáctico fue un recinto cerrado, donde el proceso de enseñanza-aprendizaje se llevó a cabo durante jornadas de seis horas continuas con un descanso intermedio. El escenario tuvo un predominio del rol docente desde la estructura académica y metodológica en la que se desarrollaron los módulos: 
La clase inicia a las 7:00 a. m. y se desarrolla en un salón. Las sillas son dispuestas por el grupo en mesa redonda, de acuerdo a lo indicado por la profesora, quien presenta la metodología en la que se desarrollará el módulo e inicia la clase con la proyección de diapositivas. Tras tres horas y media de clase, la profesora les indica que tienen treinta minutos para tomar algo y volver al salón. La jornada finaliza a la 1:15 p. m. (grupo 1, estudiantes, 3 semestre, observación 1)

La clase inicia a las 7:00 a. m. y se desarrolla en el auditorio hasta la 1:00 p. m. La docente enfrente de los estudiantes pide que ordenen el salón en filas para realizar la observación de algunos videos. Tras cuatro horas y media de clase, las profesoras les indican que pueden salir a descanso y volver en 20 minutos. (grupo 1, estudiantes, 3 semestre, observación 2)

Para Porlán \& Martín (1994), el profesor como mediador posee un papel regulador y transformador de iniciativas externas que incidan en la dinámica de las aulas (p. 18). En lo observado, se evidencia la tendencia conductista del docente en el aula, como transmisor de conocimientos en un tiempo determinado. Se deja de lado la aplicación y enseñanza de estrategias que podrían ayudar al estudiante a integrar el conocimiento del enfermero para el autocuidado, el cuidado de las personas y el mejoramiento de las relaciones interpersonales en un contexto asistencial y social (Rideout citado por Noreña et al., 2013).

En la CSP, se realizaron cuatro mapas en línea del tiempo (imágenes 1 y 2), donde el escenario didáctico es la lámpara como imagen que representa el aula de clase, que es el territorio existencial sobre el que los docentes plasmaron, desde un presente y un futuro, el significado y representación que tienen sus prácticas pedagógicas en el programa.

En la narración y el nombre en la clase, se encuentra que los docentes "no mapearon un territorio físico, sino que se adentraron en la complejidad de los territorios epistemológicos (de conocimiento) y los existenciales o de la vida misma" (Barragán, 2015, p. 270):

El salón como parte de la construcción de las prácticas pedagógicas del presente no es cómodo y solo se puede adecuar en filas o mesa redonda; desde lo que esperamos en un futuro como parte de la re-ingeniería: desaprender y aprender nuevamente. Es ver más allá del salón, salir de este. (Grupo 3, docentes)

En los salones del hoy falta comodidad. Son espacios rígidos en lo que tratamos de construir en colectivo. En un futuro queremos que estos sean abiertos y que se disponga de tecnología en donde las prácticas trasciendan a partir de la innovación. (Grupo 2, docentes)

A partir de la reflexión, se evidencia que el territorio se transforma al generar y proponer cambios desde un colectivo, que transita de una visión simplista y empírica a la complejidad:

El salón no es adecuado y es rígido. De este parte el autoconocimiento, autoconcepto, autorreconocimiento y autoestima, sobre los que se crean las prácticas del presente. En el futuro, queremos despertar solo con recuerdos del pasado en espacios libres y propicios para un desarrollo integral para el docente y el estudiante. (Grupo 3, docentes)

El salón en donde se desarrollan las prácticas pedagógicas es una jaula. No es adecuado y siempre son espacios cerrados en donde permanecemos por más de seis horas. El futuro de este lo vemos como un ecosistema que representa un aula más abierta con mayor posibilidad de asumir vivencialmente el aprender. (Grupo 4, docentes) 


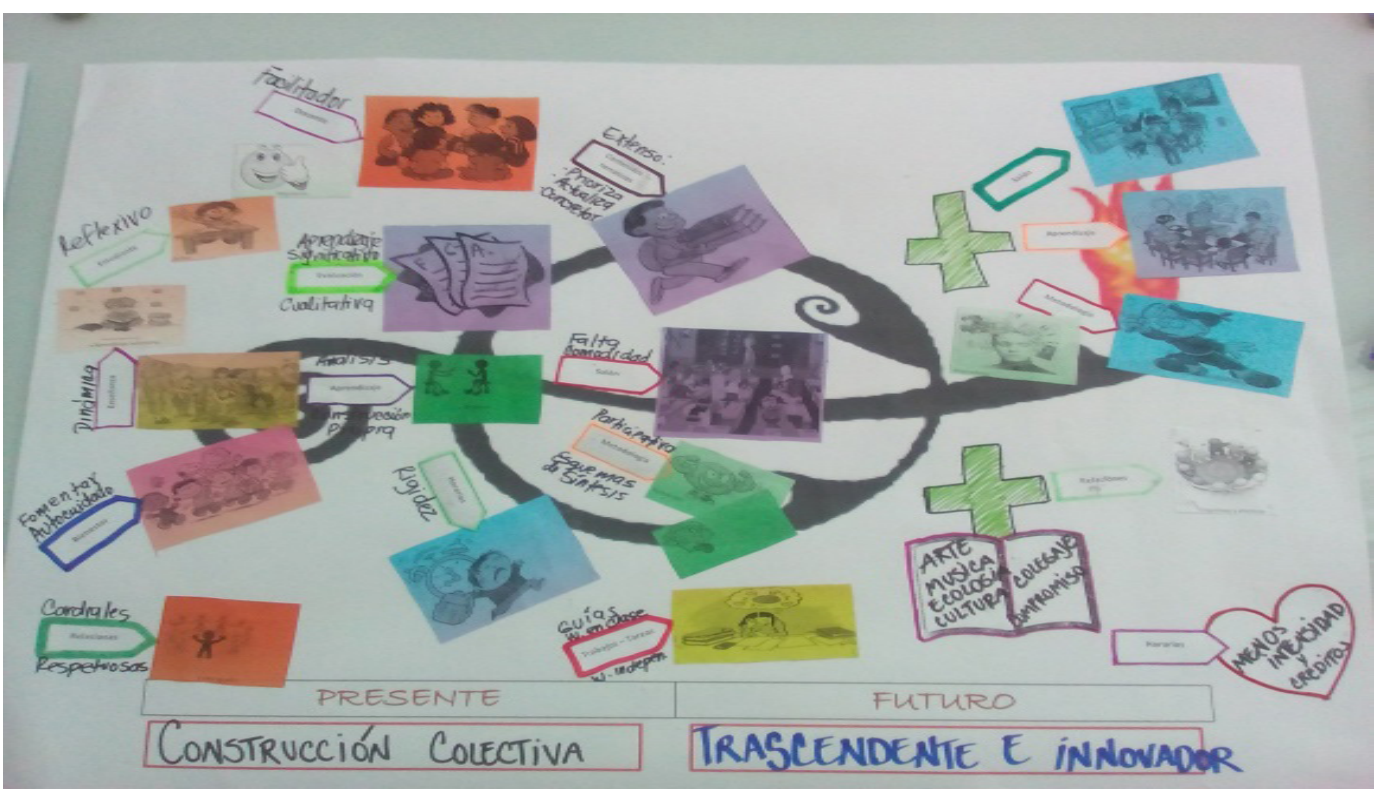

Imagen 2. Escenario didáctico: territorios epistemológicos y existenciales. Mapa: Construcción colectiva y trascendente e innovador. Fuente: docentes del programa de enfermería.

El escenario didáctico hace parte del ambiente académico, en donde confluyen factores que posibilitan o impiden la enseñanza. Se ignora la naturaleza inestable, cambiante y ambigua de los fenómenos que se producen en el aula, al continuar con esquemas de control, que esperan generar responsabilidad y disciplina en el estudiante frente a jornadas que serán turnos o escenarios y que se convertirán en servicios de salud (Tovar, Santos, Paredes \& Bermúdez, 2011).

\section{El estudiante.}

Para Benner (citada por Carrillo et al., 2013), el estudiante es un ser integral que se desarrolla con la experiencia interactiva de los demás, en la cual se establece la participación del docente como mediador de la construcción del conocimiento en simultaneidad. En la observación de campo y la CSP, se encuentra al estudiante novato o principiante, definido por Benner (2000) como aquel que aprende reglas, conocimiento básico, logra una tarea específica a la vez y sigue las instrucciones del docente paso a paso, como se evidencia a continuación:

La profesora con ayuda de la presentación en diapositivas inicia la socialización del tema. Algunos estudiantes toman nota. Ella da ejemplos de lo expuesto y pide que conformen parejas siguiendo los siguientes criterios para realizar el taller: alineación corporal y posibles alteraciones que encuentran en la pareja, la cual debe ser esquematizada y socializada en un tiempo determinado. Tras las indicaciones, el grupo inicia la realización del ejercicio y participan sustentando hallazgos de acuerdo a los criterios. (Grupo 1, estudiantes, semestre 3, observación 3).

Para Porlán (1996), la escuela y el maestro deben centrarse en el estudiante, para que pueda expresarse, participar y aprender en un clima espontáneo y natural, donde sus 
intereses fluyan desde la participación y expresión, y para que deje de lado estructuras de poder y dominación del maestro, quien reconoce al estudiante como un ser activo. Esta definición guarda relación con lo observado en las clases de sexto semestre, en donde las docentes involucran a todos los estudiantes en la construcción del conocimiento al permitir que se enfrenten a situaciones reales:

El módulo enfermedades sistémicas del adulto está a cargo de los estudiantes. Para este, las tres profesoras han asesorado a subgrupos con antelación, que desarrollarán la clase desde su iniciativa. Es así como los estudiantes disponen y realizan montaje de cada stand para dar inicio a la socialización de los temas. (Grupo 3, estudiantes, semestre 6, observación 1)

En la CSP, se presenta el mapa la Era de los autos y el despertar de las mentes con recuerdos (imágenes 3 y 4 ).

El mapa inicia con un cartel en forma de libro: "los retos del presente... respondemos a la exigencia... comprendamos la diversidad". Enseguida, el indicador estudiante muestra dos pictogramas: uno en color naranja, donde están los estudiantes dispuestos en fila observando el tablero. Luego está el ícono de rol pasivo. En relación con el primer pictograma, está la imagen de estudiantes dispuestos en mesa redonda en interacción con el docente. Desde lo expuesto por los grupos, se encuentra la siguiente representación de estudiante para el presente:

Es un ser que cuestiona si el docente sabe o no sabe. Desconocen nuestro conocimiento, experiencia y habilidades. Vemos en el estudiante una dualidad: algunos jóvenes son creativos y con buena actitud, pero también se encuentra el que quiere que todo se le dé. Es así como tal vez se convierten en títeres. (Grupo 1, docentes)

Algo que sabemos, pero no lo accionamos es que todos los estudiantes no son iguales. Son personas unitarias que solo se interesan por su bienestar y no por el de los demás. Es así como unos prefieren ser pasivos y otros, activos, pero siempre primará el confort de la mayoría. (Grupo 3, docentes)

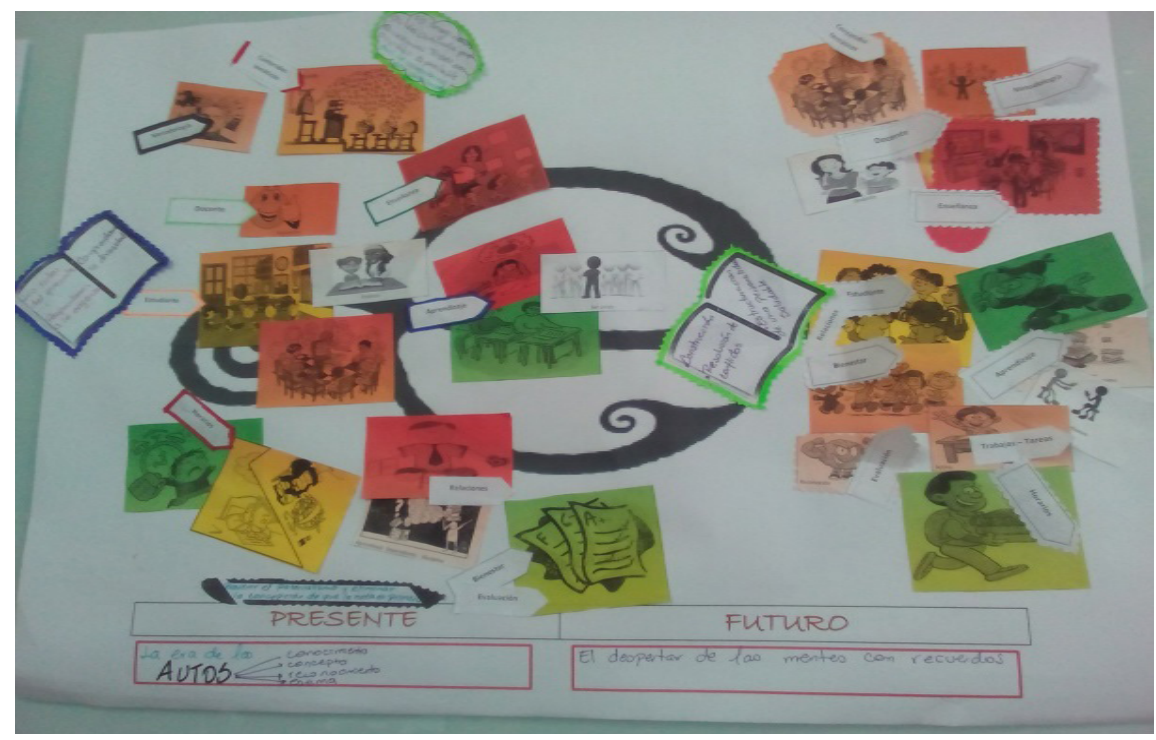

Imagen 3. Mapa: Era de los autos y el despertar de las mentes con recuerdos. Fuente: docentes del programa de enfermería. 
Los resultados expuestos guardan relación con lo observado en el aula donde el docente desarrolla sus prácticas. Allí hay dos tipos de estudiantes: pasivos o novatos y activos. Reconocer - o no- la diversidad de estudiantes, sus necesidades y fortalezas como participantes de un proceso continuo y trascendente permite la adquisición de habilidades cognitivas, afectivas, comportamentales y conductuales que dependen del proceso educativo y de la etapa en que se encuentren (Benner, 2000).

Para Porlán \& Martín (1994), antes de iniciar sus clases, los docentes deben conocer a sus estudiantes, su contexto, qué saben, qué les interesa o los motiva. Los estudiantes en re-ingeniería, desde el primer mapa, son visualizados en un futuro con los íconos de seres únicos y compañeristas. Alrededor se encuentran carteles que dicen: "excelentes personas, excelentes profesionales, líderes, ministros, presidentes, con reconocimiento profesional”.

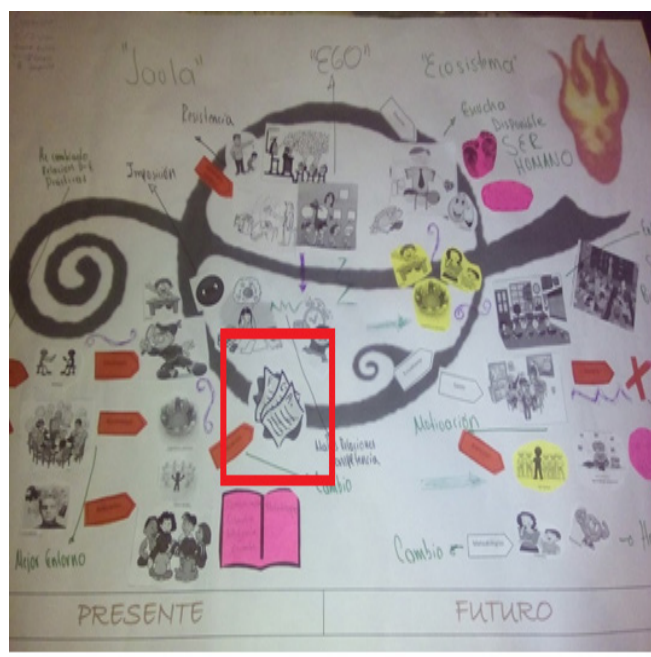

Imagen 4. Mapa: La jaula y el ecosistema. Fuente: docentes del programa de enfermería.

El mapa Desde el despertar de las mentes evidencia la idea de estudiante que esperan los docentes para el futuro: "Queremos un estudiante autónomo, seguro. Que sean excelentes personas y profesionales", "Debe ser un ser humano visto desde su entorno, reflexivo y propositivo, investigador y saludable en todas sus dimensiones" (Grupo 3, docentes).

El estudiante del futuro, según los docentes, es coherente con lo propuesto en el modelo pedagógico holístico, como proceso totalizador multifactorial en donde los actores son vistos como seres integrales que interactúan con factores que determinan el enseñar y aprender como un rasgo configuracional del mismo (Fuentes \& Álvarez, 2001).

\section{Estrategias pedagógicas.}

Las estrategias pedagógicas son elementos que orientan la práctica docente y el proceso de enseñanza-aprendizaje. Dependen del proceso de reflexión que el docente hace sobre su pertinencia, coherencia con el currículo y las necesidades particulares del estudiante (Sacristán, 2010). A partir de la observación realizada, las estrategias se pueden contrastar con la secuencia metodológica del modelo tradicional de Porlán y Martín (1994), que se estructura en tres momentos:

\section{Primer momento.}

"Explicación del profesor, relato de contenidos y conceptos siguiendo una lógica formal 
y académica, procurando que los alumnos comprendan o se enteren” (Porlán \& Martín, 1994, p. 9), lo cual guarda relación con lo observado en las dos asignaturas: "El tema del módulo es riesgo biológico y proceso infeccioso, por lo que la docente pide hacer subgrupos para socializar y aclarar los conceptos. Da a conocer que de esta manera se desarrollará la primera parte del módulo" (Grupo 1, semestre 3, observación 3).

El tema del módulo es reanimación cardio-pulmonar básica. Para su desarrollo, la profesora indica que estará bajo las guías realizadas por la AHA, por lo que se observarán videos y situaciones clínicas que ella orientará y explicará, antes de realizar la secuencia en los simuladores. (Grupo 3, semestre 6, observación1)

Durante la observación de los estudiantes de séptimo semestre, se encontró que uno de los temas del módulo no es desarrollado por los docentes, sino por ellos, quienes con la construcción de stands exponen los temas. Sin embargo, aunque es una estrategia innovadora, sigue una estructura tradicional alternativa. Según Porlán \& Martín (1994), el rol del estudiante "consiste en desarrollar un conjunto de respuestas de aprendizaje, al seguir pautas y secuencias de actividades determinadas de antemano por el profesor" (p. 10). Los estudiantes conforman los grupos y realizan preparación, programación y secuencia de actividades asesoradas por las docentes, previas a la socialización con los demás compañeros y posteriormente evaluadas:

La clase inicia con el stand del grupo uno, que socializa el tema de lupus con ayuda de diapositivas. Mientras tanto, una de las integrantes lee en las copias alguna información y precisa conceptos - en torno a fisiopatología, signos, síntomas y tratamiento- al grupo de estudiantes, que se observa dividido. Algunos están atentos y otros estudian para su exposición. Las profesoras toman nota de lo expuesto por el grupo y al finalizar se reúnen para deliberar la nota, la cual no es socializada con los estudiantes en esta sesión. (Grupo 3, semestre 6, observación 2)

La secuencia positivista y lógica de este primer momento dificulta el proceso de deconstrucción y reconstrucción del conocimiento del estudiante y del maestro como mediador - aquel que indaga, problematiza y propone concepciones sistémicas y complejas de la realidad, en autorregulación y responsabilidad de su actuar (Fuentes \& Álvarez, 2001) - Esto es reconocido y descrito por los docentes durante el taller de CSP con los siguientes relatos: "Todavía tenemos prácticas tradicionales y creemos que en este momento las estamos tratando de minimizar al máximo. Aunque la mayoría de los estudiantes no lo vean, así es" (Grupo 1, docentes). "Nuevas estrategias son una sobrecarga por el tiempo. Los estudiantes a veces pueden proponer, Estamos tratando de cambiar, pero seguimos muy ligados a esquemas repetitivos en donde lo importante es avanzar en las temáticas" (Grupo 3 , docentes).

\section{Segundo momento.}

"Realización de actividades previstas para fijar los contenidos, la mayor parte de ellas inducen a reproducir lo explicado, se trata de actividades fundamentalmente mecánicas que refuerzan la memorización" (Porlán \& Martín, 1994, p. 7). Los resultados de la observación y los expuestos en la CSP guardan relación con lo propuesto por Porlán:

La docente presenta la segunda y tercera temática del módulo - higiene postural y 
movimiento-. Para la socialización, proyecta el video El sentido del equilibrio. Los estudiantes escuchan mientras toman nota. Al finalizar el video, la profesora retroalimenta y enfatiza en los tipos de articulación que se involucran en el movimiento. Para cerrar el tema, pide al grupo que hagan cinco filas y sigan sus movimientos, reconociendo el tipo y la articulación involucrada. El grupo sigue los movimientos de la profesora, pero muchos se ríen de no lograr ejecutarlos ni reconocerlos. (Grupo 1, estudiantes, semestre 3, observación 3)

Estamos generando procesos de aprendizaje autónomo a partir de estrategias participativas como sociodrama y cosas lúdicas, en donde se observa si apropió o no el conocimiento. También contamos para cada clase con un objetivo, una metodología y una evaluación específica. (Grupo 3, docentes).

Sobre el segundo momento inciden factores que facilitan o dificultan las estrategias pedagógicas: el horario, los contenidos temáticos, trabajos y tareas; cartografiados por los docentes en los mapas, lo cual se contrastó con lo observado en el salón de clase. Como ejemplo, se presenta el mapa Construcción colectiva y Trascendente e innovador (imagen 5). El pictograma para representar los horarios está demarcado con un círculo azul. En la imagen un niño corre mientras es perseguido por un gran reloj. Junto al indicador se observa la palabra "rigidez". Este pictograma fue utilizado por los cuatro grupos para representar el tiempo en el que se realizan las estrategias pedagógicas.

En lo expuesto por los grupos, está la siguiente representación: "en esto sí somos rígidos. A pesar de que son seis horas, siguen siendo extenuantes" (Grupo 3, docentes), "El asunto de los horarios es fuerte. Complica los procesos de los estudiantes” (Grupo 2, docentes) "Son extensos y desgastantes" (Grupo 4, docentes).

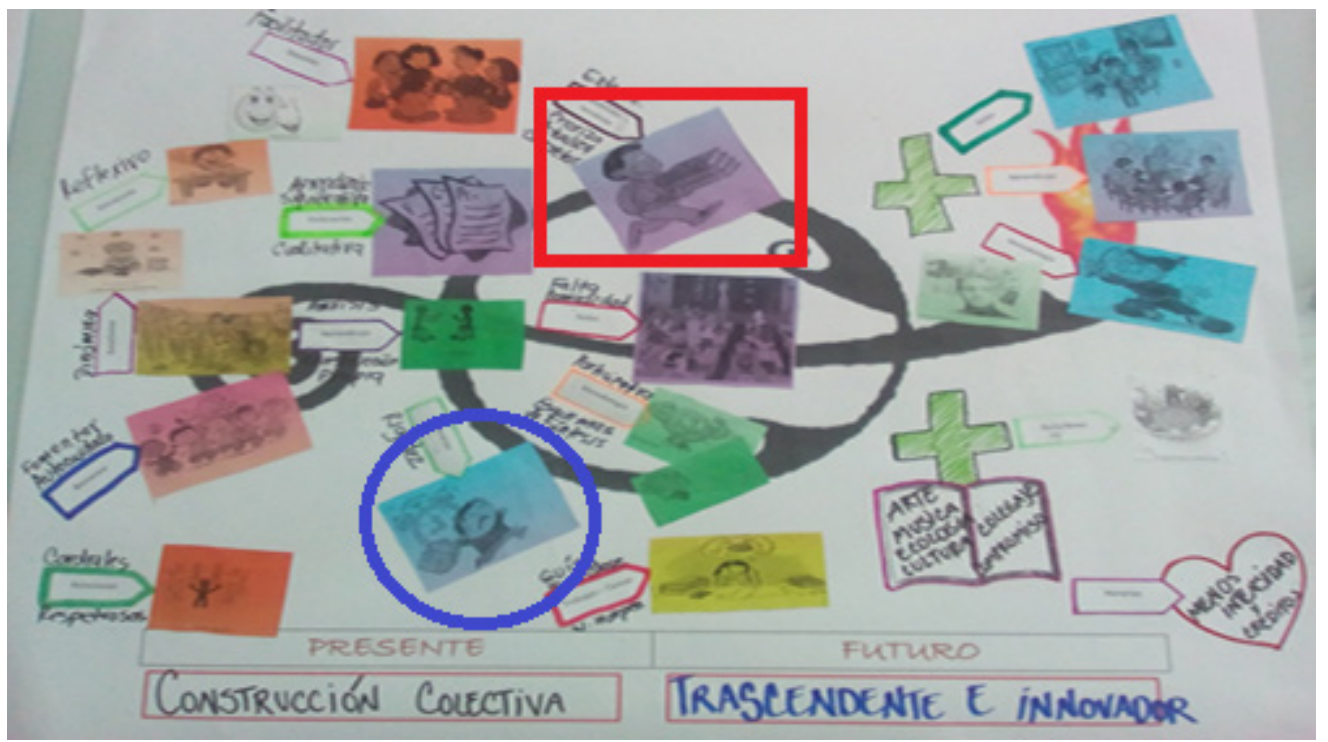

Imagen 5. Mapa: Construcción colectiva y Trascendente e innovador. Fuente: docentes del programa de enfermería.

En relación con los contenidos temáticos, el mapa presenta el pictograma de un estudiante sonriente que lleva en sus manos unos libros. Este se encuentra en la parte superior, dentro de un recuadro rojo. Cerca del indicador, está la palabra "extenso" y, bajo él, las palabras "prioriza, actualiza y concretar". Según Ospina (2006), las clases en enfermería 
se estructuran para que el estudiante adquiera un conocimiento determinado que lleva a que el docente se centre de una forma conductista en los contenidos, más que en el proceso de generar análisis, reflexión y discusión, lo cual guarda relación con lo expuesto por los docentes en la CSP: "Sabemos que es extenso, pero priorizamos en lo que es necesario para el estudiante" (grupo 3, docentes). "Los contenidos son muy largos y llevan a que el proceso de aprendizaje se vea afectado" (Grupo 1, docentes).

Son muy extensos. Así queramos innovar con estrategias y metodologías, el tiempo no da para desarrollarlas. También tenemos la concepción de que, entre más contenidos se enseñe, es mejor, así conozcamos que todo no es reflexionado de la mejor manera. (Grupo 2, docentes)

Jerez y Oyarzo (2015) concluyen que los factores estresores que inciden en el proceso de aprendizaje de los estudiantes son: la sobrecarga de tareas y el tiempo limitado para hacer trabajos, los cuales son identificados por los cuatro grupos de docentes con el cartel "trabajos y las tareas" y están representados con los pictogramas e iconogramas de la imagen 6: "Las tareas son para realizar en forma independiente y socializar en clase. Muchas veces el tiempo y el número de asignaturas no dan para que los estudiantes cumplan con todo" (grupo 3, docentes).

Los trabajos y tareas a partir de guías se manejan en casi todos los espacios de formación. Lo que no nos cuestionamos es cuántas debe hacer el estudiante por día para tratar de responder a todo y si esta es una estrategia adecuada. (grupo 4, docentes)

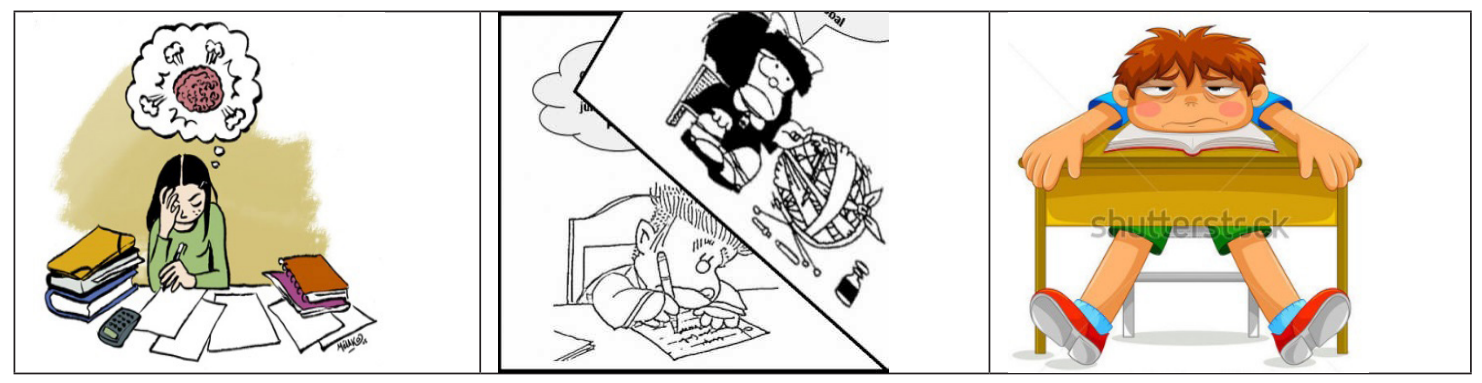

Imagen 6. Trabajo y tareas. Fuente: docentes del programa de enfermería.

Al contrastar lo evidenciado en el taller de CSP con la observación de campo, se encuentra que la guía de trabajo independiente es una estrategia de las dos asignaturas. Sin embargo, hay hallazgos que dificultan su desarrollo y socialización:

La profesora inicia la clase preguntando al grupo de estudiantes por el desarrollo de la guía de movilidad, tema a desarrollar en la sesión. El grupo contesta no haberla desarrollado. La profesora indica: "deben asumirlo para el parcial de la próxima clase". Al transcurrir el desarrollo de la clase, la profesora pide a los estudiantes que digan el nombre de los movimientos articulares que realiza, sin obtener respuesta, por lo que les dice: "el silencio es porque ustedes no desarrollaron la guía para el día de hoy". (Grupo 1, estudiantes, semestre 3 , observación 2)

En la segunda sesión del desarrollo del módulo del tercer semestre, los estudiantes retoman apuntes y algunos revisan los conceptos en el celular. Uno de los subgrupos define de forma imprecisa los conceptos que se abordan según lo referido por la docente, quien les pregunta si desarrollaron la guía. Muchos de los estudiantes permanecen en silencio. Solo algunos 
responden que sí, mientras otros murmuran que no toda, por falta de tiempo. (Grupo 3, semestre 6, observación 3)

Los factores: horarios, contenido temático, trabajos y tareas, antes presentados desde la interpretación del docente de enfermería, se sustentan desde lo teórico. Según Sacristán (2010), "el tiempo de la clase se rellena de tareas escolares y de esfuerzos por mantener un cierto orden social dentro del horario escolar, bajo una forma de interacción entre profesores y alumnos" (p. 4).

\section{Tercer momento.}

"Realización de actividades de control sobre lo aprendido, preguntas orales o pruebas escritas que se traducen en una valoración numérica de la capacidad de memorización de los alumnos" (Porlán \& Martín, 1994, p. 7). La secuencia metodológica en la que se desarrollan las estrategias pedagógicas finaliza con la evaluación de los contenidos socializados durante cada sesión y módulo:

La clase inicia a las 7 a. m. Durante la primera hora, el grupo presenta el parcial del módulo que finalizó la semana anterior. El auditorio es dispuesto en filas y cada estudiante resuelve su pliego, mientras las docentes los observan: una de ellas sentada enfrente, mientras otra camina por el salón. (grupo 1, semestre 3, observación 3)

La clase inicia a las 7 a. m. Durante las dos primeras horas, el grupo presenta el parcial programado por las docentes, por lo que el salón está dispuesto en filas. Cada estudiante responde su pliego y solo tres estudiantes piden asesoría a las profesoras para aclarar dudas frente a las preguntas que deben resolver. (grupo 3, semestre 6, observación 2)

La evaluación, como instrumento de clasificación, aprobación y cuantificación del saber, se ha mantenido en los docentes de enfermería, quienes han centrado el objetivo en la realización de pruebas y exámenes que buscan la reproducción de los contenidos transmitidos (Vasconcelos, Backes \& Gue, 2011). Lo cartografiado por los cuatro grupos de docentes se representa en el pictograma señalado dentro del rectángulo, en el mapa La jaula y el ecosistema (Imagen 4).

La socialización cobra sentido y guarda relación con lo observado con los siguientes relatos: "la evaluación es tradicional y se lleva con esta a que los estudiantes sean competentes y se centren solo en los logros académicos y en la nota" (Grupo 4, docentes). "El aprendizaje sí está centrado en la nota, en pasar la evaluación. Por eso, es la era del auto: autoconcepto y reconocimiento" (Grupo 4, docentes).

En el modelo pedagógico del programa, la evaluación se establece como una estrategia democrática que se construye entre estudiantes y docentes y que se integra al proceso de enseñanza aprendizaje, es holística, integradora y permanente y no una actividad final frente a los resultados. Esta concepción no concuerda con los resultados expuestos, pero sí se acerca a la perspectiva que tienen los docentes sobre la evaluación a realizar en un futuro, como se presenta a continuación: "La evaluación debe ser más que una calificación. Debe ser un proceso de retroalimentación" (Grupo 4, docentes). "Que esta sea en torno a un aprendizaje significativo, no tanto de memoria, ni por una nota, sino por la capacidad de reflexionar" (Grupo 3, docentes). 
El taller de CSP facilitó la reflexión y construcción de posibilidades a futuro en torno a las estrategias pedagógicas de los docentes, desde propuestas de transformaciones: "Queremos innovar desde la tecnología, para que el aprendizaje pueda ser un proceso reflexivo y dinámico" (Grupo 1, docentes). "Queremos unas estrategias trascendentes e innovadoras, desde la investigación y desde la creación del conocimiento del estudiante; que se incluya el arte, la música y la ecología como parte de la enseñanza del cuidado" (grupo 2, docentes).

\section{Conclusiones}

El mediador y el enseñar, como categorías, permitieron identificar las prácticas pedagógicas de los docentes, que siguen el patrón propuesto por Melis (citado por Carranza, 2005), quien dice que "el profesor de enfermería es el poseedor del conocimiento, y por tanto, la metodología y estrategia aplicada en la enseñanza es tanto positivista como racionalista o empírico analítica" (p. 17), lo que dificulta la relación y coherencia con el modelo constructivista, que espera que el educador establezca una relación con la teoría crítica, al abordar elementos que faciliten la comprensión de una enfermería socio- crítica, hacia un paradigma emancipador del cuidado, desde una práctica reflexiva y el pensamiento crítico como base para su desarrollo (Sánchez, Aguayo \& Galmedes, 2017).

El maestro de enfermería del presente debe transitar y superar la prolongación de un concepto hegemónico que se ha adaptado a través del tiempo desde el modelo conductista $\mathrm{y}$ tradicional, donde el docente transmite conocimientos y el estudiante asume un rol pasivo frente al conocimiento que es ofrecido y considerado útil para su desempeño laboral. Para García, Siles, Martínez, Martínez y Álvarez (2017), se requiere que los currículos adopten metodologías más flexibles para permitir a los estudiantes ser protagonistas y participantes activos de sus propios procesos de aprendizaje

El logro de la relación entre el maestro expuesto en el modelo pedagógico y el identificado está en la propuesta del maestro del futuro, como aquel ser humano mediador e investigador en el aula, que facilita el desarrollo de un ser activo, crítico y propositivo, desde procesos cotidianos y reflexivos de la práctica que desarrolla cotidianamente (Rubio \& Arias, 2013).

Se promueve la reflexión de una nueva forma de comprender y proponer en el aula, como escenario didáctico, donde el entorno y las relaciones hagan parte de prácticas coherentes que trasciendan e involucren el proceso de enseñanza-aprendizaje, como un fin transpersonal y de reciprocidad: docente-estudiante-contexto y sociedad. De igual manera, el docente mediador requiere integrar la relación entre la teoría y la praxis desde un abordaje complejo, ya que, mientras no se articule el conocimiento teórico con el contexto de la naturaleza de la práctica, será difícil cerrar la brecha existente entre las dos (Piedrahita \& Rosero, 2017).

Aprender a cuidar ocurre necesariamente en la relación entre el docente-estudiante y la persona cuidada, donde el estudiante aplica y trasforma los conocimientos teóricos aprendidos en el aula, a través de acciones prácticas de cuidado, las cuales son apoyadas a partir de comunicaciones afectivas y metodologías integrativas (Bettancourt, Muñoz, Barbosa \& Fernandes, 2011).

Para González y Duque (2019), las prácticas pedagógicas tradicionales evidencian un 
estudiante que continúa pasivo y difiere con la concepción del individuo que se desea formar desde un modelo pedagógico. Se requiere el cambio para que este emerja como un ser activo, a quien se le permite crecer a su tiempo, reconocer su independencia, tener tolerancia, con honestidad y sinceridad, un estudiante cuyo maestro cree en su potencial como una condición necesaria para el aprendizaje.

Las estrategias pedagógicas guardan relación con el proceso reflexivo que el docente hace de sus prácticas y la coherencia con el modelo pedagógico, las cuales discrepan del enfoque crítico-constructivista que promueve la formación en enfermería, al reproducir estrategias convencionales y verticales, bajo modelos tradicionales que homogenizan y minimizan la diversidad de las prácticas (Sacristán, 1995). Esto conduce a que el sentido de la acción pedagógica no sea evidente a la luz del ser humano complejo, en constante transformación y en búsqueda de la trascendencia.

Los resultados del presente estudio describen el significado que emerge de los docentes al identificar sus prácticas pedagógicas. Asimismo, brindan herramientas para que el docente de enfermería, reconozca su quehacer, la experiencia y las necesidades de aprendizaje de los estudiantes, que les permitan reflexionar sobre su práctica y la coherencia con el modelo constructivista del programa.

\section{Referencias}

Agama, A., \& Crespo, S. (2016). Modelo constructivista y tradicional: influencia sobre el aprendizaje, estructuración del conocimiento y motivación en alumnos de enfermería. Index de Enfermería, 25(1-2), 109-113.

Barragán, D. (2015). La cartografía social-pedagógica: entre teoría y metodología. Revista ColombianadeEducación,(70),247-285.https://doi.org/10.17227/01203916.70rce247.285

Barragán, D., \& Amador, C. (2014). La cartografía social-pedagógica: una oportunidad para producir conocimiento y repensarla educación. Itinerario Educativo, (64), 127-141. https://doi.org/10.21500/01212753.1422

Benner, P. (2000). The Wisdom of Our Practice. American Journal of Nursing, 100(10), 99105. https://doi.org/10.1097/00000446-200010000-00075

Bettancourt, L., Muñoz, L., Barbosa, M., \& Fernandes, M. (2011). El docente de enfermería en los campos de práctica clínica: un enfoque fenomenológico. Rev. Latino-Am. Enfermagem, 19(5), 1-9.

Burns, N., \& Grove, S. (2004). Investigación en Enfermería: Introducción a la investigación cualitativa. Elsevier, 17(3), 385-430.

Carranza, A. (2005). El constructivismo como estrategia educativa: formación profesional en enfermería. Revistas enfermería, (30), 15-21.

Carrillo, A., García, L., Wilches, N., Cárdenas, C., \& Díaz, I. (2013). La filosofía de Patricia Benner y la práctica clínica. Revista Enfermería Global, 12(32), 346-361. https://doi. org/10.6018/eglobal.12.4.151581 
Castro, M., \& Pereira, W. (2011). Integral care: conceptions and practices of nursing professors. Revista Brasileira de Enfermagem, 64(3), 486-493.

Cadavid, A., \& Calderón, I. (2001). Análisis del concepto enseñanza en las teorías curriculares de Lawrence Stenhouse y José Gimeno Sacristán. Revista educación y pedagogía, 16(40), 143-151.

Flórez, C., Mayorga, A., \& Vargas, L. (2017). La práctica formativa como escenario de aprendizaje significativo del cuidado de enfermería. Rev. Colomb. Enferm, 12(14), 93-10. https://doi.org/10.18270/rce.v12i14.2031

Fuentes, H., \& Álvarez, I. (2001). La formación por la contemporaneidad, modelo holístico configuracional de la didáctica de la educación superior. Esquemas pedagógicos, 3, 3-20.

García, B., Siles, G., Martínez, M., Martínez, B., \& Álvarez, B. (2017). Metodologías de enseñanza-aprendizaje en enfermería: ¿Es el portafolio una metodología acorde con el Espacio Europeo de Educación Superior?. Enfermería Docente, 108, 24-28.

García, J. (2005). El pensamiento pedagógico de José Martí acerca de la formación docente y el currículo educativo. Educación, 2(2), 67-75. https://doi.org/10.15517/revedu. v29i2.2240

González, L., \& Duque, P. (2019). Prácticas pedagógicas: una mirada a la formación profesional de enfermería. Revista de Investigaciones UCM, 19(33), 91-102.

Jaramillo, L., \& Aguirre, J. (2012). Aportes del método fenomenológico a la investigación educativa. Revista Latinoamericana de Estudios Educativos, 8(2), 51-74.

Jerez, M., \& Oyarzo, C. (2015). Estrés académico en estudiantes del Departamento de Salud de la Universidad de Los Lagos Osorno. Revista chilena de neuro-psiquiatría, 53(3), 149157. https://dx.doi.org/10.4067/S0717-92272015000300002.

Noreña, A., Alcaraz, N., Amador, G., \& Ramos, F., (2013). La relación de ayuda en el campo de la enseñanza-aprendizaje. Cultura de los cuidados, 17(35), 106-114. https://doi. org/10.7184/cuid.2013.35.10

Ospina, A. (2006). Currículo por competencias en la Universidad de la Sabana. Aquichán, 6(1), 117-124.

Paim, S., Iappe, T., \& Rocha, D. (2015). Métodos de enseñanza utilizados por docentes del curso de enfermería: enfoque en la metodología de investigación. Enfermería Global, 14(37), 136-152. https://doi.org/10.6018/eglobal.14.1.186291

Piedrahita, L., \& Rosero, A. (2017). Relación entre teoría y praxis en la formación de profesionales de enfermería: revisión integradora de literatura. Enfermería Global, 16(47), 679-706. https://dx.doi.org/10.6018/eglobal.16.3.269261.

Porlán, R. (1987). El maestro como investigador en el aula, investigar para conocer, conocer para enseñar. Investigación en la escuela, (1), 63-69.

Porlán, R. (1996). Cambiar la escuela. Buenos Aires: Magisterio del Río de la Plata.

Porlán, R., \& Martín, J. (1994). El diario del profesor, un recurso para la investigación en el aula. Investigación y enseñanza, 18 -78. 
Prado, M., Souza, M., Monticelli, M., Cometto, M., \& Gómez, F. (2013). Investigación cualitativa en enfermería, metodología y didáctica. Washington, D. C .: Organización Panamericana de la Salud.

República de Colombia. Ministerio de Educación. (2004) “Ley 911 de 2004”. Diario Oficial. No. 45.693 de 6 de octubre de 2004.

Rodrigues, M., Mendes, J., \& Carvalho, J. (2008). Educational obstacles in the everyday living of the nurse teacher's pedagogical practice. Revista Brasileira de Enfermagem, 61(4), 435440.

Rubio, M., \& Arias, M. (2013). Fenomenología y conocimiento disciplinar de enfermería. Revista Cubana de Enfermería, 29(3), 191-198.

Sacristán, J. (1991). El currículum. Una reflexión sobre la práctica. Madrid. Morata.

Sacristán, J. (1995). Diversos y también desiguales. Qué hacer en educación. En Kiriki, (38), pp. 19-25.

Sacristán, J. (2010). La carrera profesional para el profesorado. Revista Interuniversitaria de Formación del Profesorado, 68(24,2), 243-260.

Sánchez, B. (2001). La experiencia de ser cuidadora de una persona en situación de enfermedad crónica. Revista Investigación y educación en enfermería, 19(2), 36-59.

Sánchez, J., Aguayo, C., \& Galdames, C. (2017). Desarrollo del conocimiento de enfermería, en busca del cuidado profesional. Relación con la teoría critica. Revista Cubana De Enfermería, 33(3), 1-18.

Stake, R. (2007). Investigación con estudio de caso (4ª ed.). Madrid: Ediciones Morata.

Tovar, M., Santos, M., Paredes, S., \& Bermúdez, A. (2013). El constructivismo y la formación profesional de Enfermería. Biblioteca Las Casas, 9(2), 1-11.

Vasconcelos, C., Backes, V., \& Gue, J. (2011). La evaluación en la enseñanza de grado en enfermería en América Latina: una revisión integrativa. Revista Enfermería Global, 10(23), 96-117. https://doi.org/10.4321/s1695-61412011000300008 